\title{
Magnetic-tip trap system
}

\author{
Oki Gunawan $\odot,{ }^{1, *}$ Jason Kristiano $\odot,{ }^{1,2}$ and Hendra Kwee $\odot^{2,3}$ \\ ${ }^{1}$ IBM T. J. Watson Research Center, Yorktown Heights, New York 10598, USA \\ ${ }^{2}$ Simetri Foundation, Tangerang, Indonesia 15334 \\ ${ }^{3}$ Surya College of Education, Tangerang, Indonesia 15115
}

(Received 22 April 2019; revised manuscript received 14 June 2019; accepted 15 February 2020; published 23 March 2020)

\begin{abstract}
We report a detailed theoretical model of a recently demonstrated magnetic trap system based on a pair of magnetic tips. The model takes into account key parameters such as tip diameter, facet angle, and gap separation. It yields quantitative descriptions consistent with experiments such as the vertical and radial frequency, equilibrium position, and the optimum facet angle that produces the strongest confinement. We arrive at the striking conclusion that a maximum confinement enhancement can be achieved at an optimum facet angle $\theta_{\max }=\arccos \sqrt{2 / 3}$. We also discuss a critical gap which is the maximum gap between the tips for the confinement enhancement effect to occur. This magnetic trap and its theoretical model serve as an interesting example of a simple and elementary magnetic trap in physics.
\end{abstract}

DOI: 10.1103/PhysRevResearch.2.013359

\section{INTRODUCTION}

Various electromagnetic trap systems play important roles in physics for their ability to trap and isolate particles or matter and have produced many applications and discoveries. Examples are the Penning trap [1,2], optical dipole trap or optical tweezer [3-5], magneto-optic trap [6,7], and various diamagnetic traps [8-12]. For diamagnetic trap systems, a high field-gradient product $(B \nabla B)$ is necessary to achieve trapping or levitation [13]. A new approach is to use magnetictip geometry as recently demonstrated by O'Brien et al. [14]. The tip geometry maximizes $B \nabla B$ at the trapped object, which leads to stronger field confinement and thus high frequency and a high quality factor $(Q)$. These characteristics are of high interest for research that explores macroscopic limits of classical mechanics and quantum mechanics. Such a magnetic trap also offers an interesting alternative to the optical trap as the latter can lead to excessive heating and encounters instabilities in vacuum [15]. The ability to achieve high magnetic field gradients in a localized position using the magnetic tip is also useful for other applications such as for nuclear magnetic resonant imaging [16] and magnetic force microscopy [17].

Currently, there is strong interest in magnetic trap systems for various applications such as precision gravimetry [18], study of displacement and velocity of Brownian particles [19], gas temperature measurement [20], and research that explores the boundaries of the classical and quantum systems. For example, trapped nanodiamonds can be used to investigate quantum mechanical properties such as superposition

\footnotetext{
*ogunawa@us.ibm.com

Published by the American Physical Society under the terms of the Creative Commons Attribution 4.0 International license. Further distribution of this work must maintain attribution to the author(s) and the published article's title, journal citation, and DOI.
}

of states [21,22], control of electron spin of nanodiamond nitrogen-vacancy centers, and observation of electron-spinresonance properties [23]. Such a trap could also serve an important role to test quantum mechanical properties of gravity [24,25].

In a recent demonstration of a magnetic-tip trap, O'Brien et al. uses two cylindrical magnets with sharpened tips and a microdiamond as the trapped object [14]. The tips are separated by a gap $d=2 a$ as shown in Fig. 1(a). The trapping occurs due to diamagnetic repulsion that balances the gravity of the diamond and the cylindrical symmetry that produces a stable potential confinement in three dimensions. The study in Ref. [14] has reported many important physical characteristics of the trap such as the vertical and radial trap frequency, damping factor, and maximum field confinement at a certain facet angle. However, the detailed field and potential distribution of the magnetic trap have not been presented.

In this paper we present a theoretical model that provides an analytical solution of the magnetic field of the trap along its principal axis $(z)$. The model leads to rich descriptions such as equilibrium height, axial and radial oscillation frequency, the optimum facet angle of the tip to achieve the "confinement enhancement," and the "critical gap" beyond which such effect no longer applies. Beyond the recent interest in various magnetic traps, this model also serves as an elementary example of a simple magnetic trap system based on conical tip geometry. This adds to the collection of various types of diamagnetic trap systems that have been known in physics [8-12,26,27].

\section{THEORY}

We present a theoretical model of a pair of magnetic tips system as shown in Fig. 1(a). Each magnetic tip consists of a cylindrical segment of semi-infinite length and a conical segment. The magnet has a uniform volume magnetization M parallel to the cylindrical axis, however the two tips have opposing magnetization. The cylindrical segment has a radius 
$R$ and the conical tip has a facet angle $\theta$ [28]. The magnetic trap has a gap opening $d=2 a$. A diamagnetic object such as a diamond bead can be trapped or levitates near the center of the trap at equilibrium position $z_{0}$.

We first consider the magnetic field along the principal axis $z$ due to the upper magnetic tip. We can calculate the magnetic field by integrating the field contributions due to bound surface current $\mathbf{K}_{\mathbf{b}}=\mathbf{M} \times \hat{\mathbf{n}}$ all around the conical and the cylindrical segments, where $\hat{\mathbf{n}}$ is the normal of the surface element (see Appendix A for detailed calculations). Interestingly this leads to a closed-form solution. The magnetic field due to the upper magnetic tip is given as

$$
\begin{aligned}
\mathbf{B}_{\mathbf{U}}(z)= & -\frac{\mu_{0} M}{2}\left[\cos ^{2} \theta[1-\sin \theta \operatorname{arctanh}(\sin \theta)]+\cos ^{2} \theta \sin \theta \operatorname{arctanh}\left(\frac{R+(a-z) \sin \theta \cos \theta}{\sqrt{R^{2}+(a-z)^{2} \cos ^{2} \theta+R(a-z) \sin 2 \theta}}\right)\right. \\
& \left.+\frac{z-a-R \tan \theta}{\sqrt{R^{2}+(a-z+R \tan \theta)^{2}}}+\frac{(a-z) \sin ^{2} \theta \cos \theta-R \cos 2 \theta \sin \theta}{\sqrt{R^{2}+(a-z)^{2} \cos ^{2} \theta+R(a-z) \sin 2 \theta}}\right] \hat{\mathbf{z} .}
\end{aligned}
$$

By exploiting the symmetry of the problem, the total magnetic field due to the upper and lower tips is given as

$$
\mathbf{B}_{\mathbf{T}}(z)=\mathbf{B}_{\mathbf{U}}(z)-\mathbf{B}_{\mathbf{U}}(-z) .
$$

An example of the total magnetic field plot along the $z$ axis (for $R=1 \mathrm{~mm}$ and $a=15 \mu \mathrm{m}$ and $\theta=35^{\circ}$ ) is given in Fig. 1(b). We observe that the field distribution near the center of the trap is approximately linear, which leads to a harmonic potential trap. The total potential (per unit volume) of the trapped object due to magnetic interaction and gravity is given as

$$
U_{T}(z)=-\frac{\chi}{2 \mu_{0}} B_{T}^{2}(z)+\rho g z,
$$

where $\rho$ is the density of the trapped object, $\chi$ is the magnetic susceptibility, $g$ is the gravitational acceleration, and $\mu_{0}$ is the magnetic permeability in vacuum. We note that for a spherical diamagnetic object we should replace $\chi / 2$ with $\chi /(2+\chi)$ [29], however the former is a good approximation for very small $\chi$ as in the case of many diamagnetic materials (with the exception of a superconductor where $\chi=-1$ ). For the magnetic-tip trap that we use in this paper [14], the magnet is made of $\mathrm{NdFeB}$ with volume magnetization $M=10^{6} \mathrm{~A} / \mathrm{m}$, microdiamond as the trapped object with $\chi=-2.2 \times 10^{-5}$ [30], $\rho=3513 \mathrm{~kg} / \mathrm{m}^{3}$ [31], $R=1 \mathrm{~mm}$,
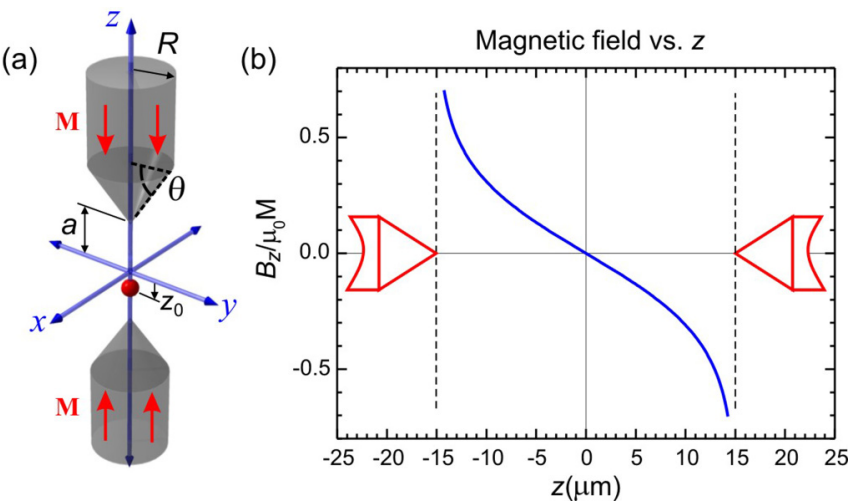

FIG. 1. (a) Geometry of the magnetic-tip trap with a trapped diamond near the center. (b) Field distribution along the $z$ axis for the "reference magnetic trap" with $\theta=35^{\circ}$ (see text). and $a=15 \mu \mathrm{m}$. We refer to this setup as the "reference magnetic trap" in this paper. For further analysis we define the "feature length" of the magnetic trap, given as $\lambda_{0}=$ $|\chi| \mu_{0} M^{2} / \rho g$, which indicates the "strength" of the magnetic trap relative to gravity. Larger $\lambda_{0}$ means a stronger magnetic trap where the diamagnetic repulsion force that equals to gravity occurs at larger distance. $\lambda_{0}$ can be derived from a simple model of a diamagnet above a spherical magnet as described in Appendix E. For the reference magnetic trap here we have $\lambda_{0}=805 \mu \mathrm{m}$.

\section{ANALYSES AND RESULTS}

We now perform the analysis in the limit of a very strong trap (i.e., $\lambda_{0} \gg a$ ) where the diamagnetic object will be trapped near the center $\left(z_{0} \sim 0\right)$. As the magnetic field is reasonably linear at the center of the trap [Fig. 1(b)], the potential can be well approximated by harmonic potential model $U_{T}(z) \approx k_{z} z^{2} / 2$, where $k_{z}$ is the "spring constant" given as $k_{z}=\partial^{2} U_{T} / \partial z^{2}$. We can obtain a very compact expression for the potential spring constant $k_{z}$ (per unit volume of the trapped object) in the strong magnetic trap limit where $z_{0} \sim 0$ :

$$
k_{z}=-\frac{\chi \mu_{0} M^{2} R^{4} \cos ^{6} \theta(a+R \tan \theta)^{2}}{a^{2}\left(R^{2}+a^{2} \cos ^{2} \theta+a R \sin 2 \theta\right)^{3}} .
$$

We show that this theoretical model provides rich descriptions of the magnetic trap characteristics that yields reasonable agreement with the experimental observation [14]. First, the model allows us to calculate the natural frequency of the vertical oscillation of the trapped object: $f_{z}=\sqrt{k_{z} / \rho} / 2 \pi$, which yields $f_{z}=348 \mathrm{~Hz}$. This is within the range of the reported frequency of $f_{z}=323$ to $411 \mathrm{~Hz}$, at pressure of 760 and 0.16 Torr, respectively [14]. Note that the observed trapped frequency at room pressure (760 Torr) is lower due to significant damping effect. Equation (4) predicts frequency that diverges as the gap decreases; in reality the smallest gap is determined by the size of the trapped particle, space for harmonic and Brownian motion of the particle, and space for optical detection. For example, the smallest nanodiamonds used in a trapping study are in the range of 20-500 $\mathrm{nm}$ [32]. Thus practically the smallest gap cannot be less than $1 \mu \mathrm{m}$. As 
a reference, the frequency of a magnetic-tip trap with $1-\mu \mathrm{m}$ gap for this reference magnetic trap is $10.9 \mathrm{kHz}$.

Second, we can calculate the equilibrium position of the trapped object that yields $z_{0}=-\rho g / k_{z}=-2.1 \mu \mathrm{m}$, which is small compared to the gap $d=30 \mu \mathrm{m}$; in other words the object (microdiamond) remains near the center of the trap as observed [14]. We note that, theoretically, stable levitation always exists irrespective of the gap due to the magnetic field characteristics that diverge near the tip as shown in Fig. 1(b). When the gap is large, the object will levitate lower until it is balanced by the diamagnetic repulsion force, which is proportional to $B d B / d z$. However, due to the finite size, the diamagnetic object will eventually touch the lower tip when the gap is large.

Third, the spring constant $k_{z}$ or the vertical trap frequency $f_{z}$ depends on the half gap $a$, the magnet cylindrical radius $R$, and the facet angle $\theta$. For a given $a$ and $R$, a maximum frequency can be achieved at an optimum facet angle $\theta_{\max }$. We can calculate this optimum facet angle, which is given as (see Appendix B)

$$
\theta_{\max }=\arccos \sqrt{\frac{2 R^{2}\left(2 a^{2}+2 \sqrt{2} a R+3 R^{2}\right)}{4 a^{4}+4 a^{2} R^{2}+9 R^{4}}} .
$$

We note that in the strong magnetic trap limit $\left(\lambda_{0}>a\right)$ the optimum facet angle depends only on the geometrical factor of the magnetic tip, i.e., $a / R$ and none of the physical properties of the magnet or the trapped object $(M, \chi, \rho)$. For the reference magnetic trap here, we obtain $\theta_{\max }=34.7^{\circ}$, which is quite close to the reported value based on numerical calculation of the boundary integral method for flat facet tips that yields $\theta_{\max }=28^{\circ}$ [14]. In the limit of a very small gap $\left(a \ll R, \lambda_{0}\right)$, Eq. (5) reduces to a very simple expression: $\theta_{\max }=\arccos \sqrt{2 / 3}=35.3^{\circ}$. This result is striking as this result does not depend on the geometry of the magnetic tip ( $R$ and $a$ ) and serves as a very simple guideline in designing the magnetic-tip trap.

Fourth, the experimental study also reported the frequency of the horizontal (radial) oscillation mode, which is half of the vertical frequency, i.e., $f_{x}=f_{y}=f_{z} / 2=198 \mathrm{~Hz}$ [14]. This is a special characteristic of this magnetic-tip trap that occurs due to cylindrical symmetry and the fact that the magnetic field is nearly linear along the $z$ axis at the center of the trap. The characteristic that $f_{r} / f_{z}=1 / 2$ can be derived using divergence-free $(\nabla \cdot \mathbf{B}=0)$ and curl-free $(\nabla \times \mathbf{B}=0)$ properties of the magnetic field and considering second-order expansion of the radial $\left(B_{r}\right)$ and axial $\left(B_{z}\right)$ components of the field in the cylindrical coordinate as described in Appendix $C$ [33].

We now extend our analysis to the impact of a wide range of geometrical and physical parameters $(a, R, \theta, \chi, \rho, M)$ on the trap characteristics. We perform numerical calculation to obtain the vertical trap frequency which represents the strength of the trap confinement. First we start with the reference magnetic trap $\left(\lambda_{0}=805 \mu \mathrm{m}\right)$ and calculate the equilibrium position $z_{0}$ of the trapped object in the magnetic trap by numerically solving $\partial U_{T} / \partial z=0$, and then we calculate the spring constant $k_{z}$ at that position $\left(z_{0}\right)$. The equilibrium position $z_{0}$ as a function of half gap $a$ and facet angle $\theta$ is plotted in Fig. 2(a). We observe that for a fixed $a$ value we achieve maximum $z_{0}$ at $\theta \approx 35^{\circ}$ (black circles). This maximum behavior apparently applies to all values of $a / R$, which implies that the magnetic trap always yields a higher levitation position $z_{0}$ at an optimum facet angle $\theta_{\max }$. Next we study the levitation height $z_{0}$ behavior with respect to half gap $a$ at varying strength of the magnetic trap $\left(\lambda_{0}=\right.$ $0.1 R, R$, and $10 R$ ) as shown in Fig. 2(c). We observe that the strongest magnetic trap (i.e., $\lambda_{0}=10 R$ ) yields the highest levitation height $z_{0}$ compared to the others $\left(\lambda_{0}=R\right.$ and $\left.0.1 R\right)$ at any $a$. For the magnetic trap with $\lambda_{0}=10 R$ at small gap $(a / R<0.1)$ the levitation height $z_{0}$ is near zero (near the center of the trap) and it starts to drop off at large gaps where $a / R>0.1$.

Next, after obtaining $z_{0}$ in Fig. 2(a) we now numerically calculate the trap frequency $f_{z}=\sqrt{k_{z} / \rho} / 2 \pi$ as a function of $a, \theta$, and $z_{0}$ and plot it in Fig. 2(b). First we observe a behavior similar to Fig. 2 (a) for a small gap $(a / R<0.07)$, i.e., the magnetic trap achieves a maximum frequency at $\theta_{\max }$ due to the confinement enhancement effect of the magnetic tip. This plot resembles the plot from numerical computation in Fig. 1(b) of Ref. [14]. However, we also observe an interesting behavior: for a large gap beyond the "critical value" $a_{c}$ this confinement enhancement effect no longer applies; i.e., when we plot $f_{z}$ vs $\theta$ at constant $a$, there is now a minimum near $\theta_{\max }$ instead of a maximum. For the magnetic trap with a very large gap $\left(a>a_{c}\right)$, to achieve high frequency one can use a low or high facet angle that corresponds to no tip or a very sharp tip, respectively. The latter is not desirable as the sharp tip will be very fragile. In practice we want to use a smaller gap $\left(a<a_{c}\right)$ to achieve a higher frequency, but not too small to provide some space for the trapped object and to allow optical detection.

Finally we study the dependence of this critical half gap $a_{c}$ with respect to varying strength of the magnetic trap by repeating the analysis in Figs. 2(a) and 2(b) at different values of $\lambda_{0}$. The result is shown in Fig. 2(d). We calculate $a_{c}$ using numerical calculations by finding the value $a$ where $\partial^{2} f_{z} / \partial \theta^{2}=0$, the data of which are shown as red points in Fig. 2(d). We observe a reasonable trend that the critical half gap $a_{c}$ increases monotonically with increasing strength of the magnetic trap (or $\lambda_{0}$ ).

Furthermore we have also attempted to derive the theoretical relationship of this critical gap as a function of $\lambda_{0}$ using series expansion of $k_{z}$ as a function of $z$ up to the fourth order evaluated at the equilibrium point $z_{0}$. Then we calculated the critical gap as detailed in Appendix D. We arrive at a simple relationship:

$$
\frac{a_{c}}{R}=\frac{\lambda_{0}\left(\sqrt{129.9 R^{2}+3.74 \lambda_{0}^{2}}-\lambda_{0}\right)}{153.86 R^{2}+3.24 \lambda_{0}^{2}} .
$$

This relationship allows us to quickly estimate the critical gap below which the confinement enhancement effect still applies in this magnetic-tip trap. However, we also note that for a strong magnetic trap $\left(\lambda_{0} / R>2\right)$ discrepancy occurs between the theoretical model and the numerical calculation due to higher orders of $a$ that were omitted in the theoretical model in Eq. (6). We also note that for practical purposes the optimum 

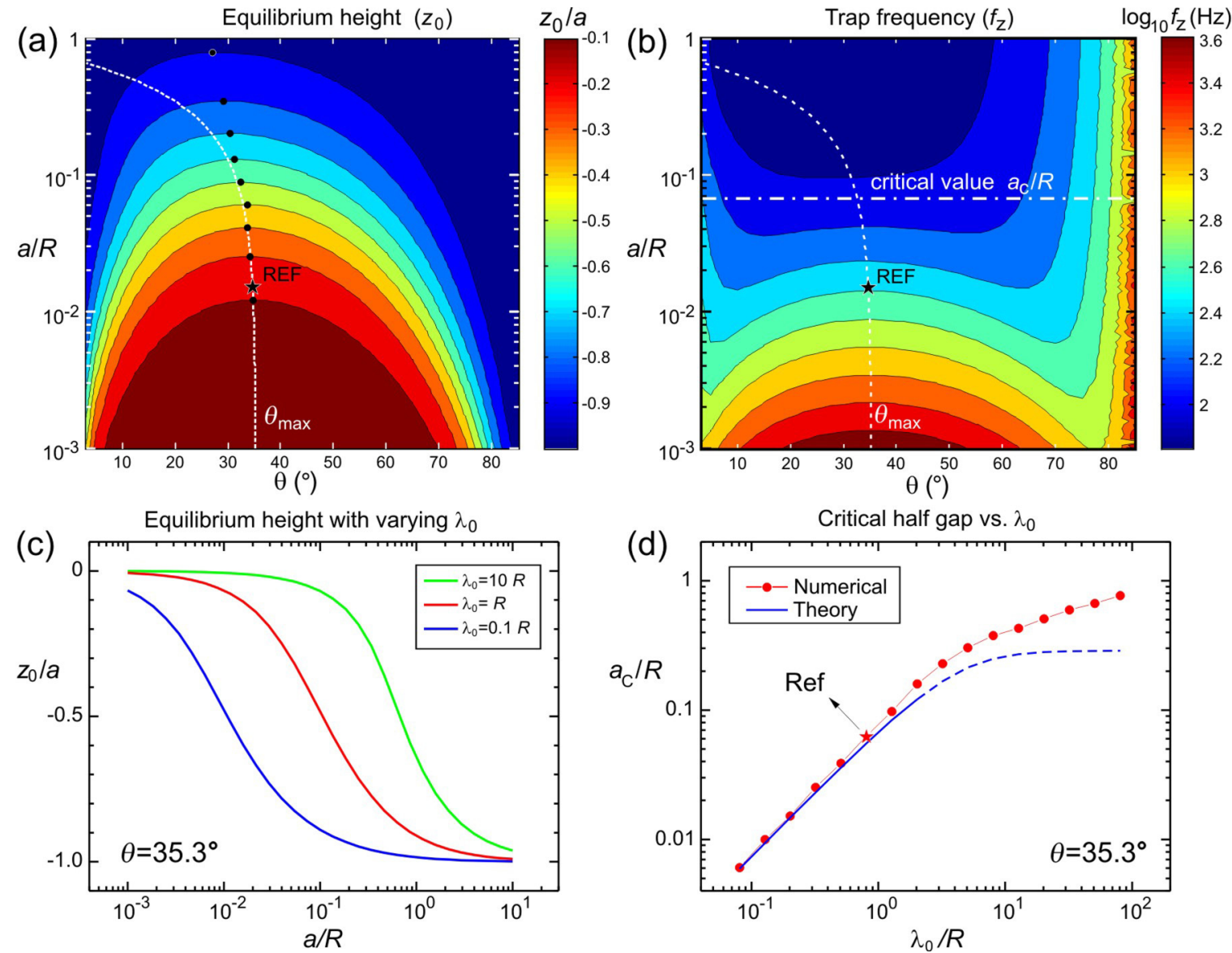

FIG. 2. (a) The equilibrium height $\left(z_{0}\right)$ dependence on the half gap $(a)$ and the facet angle $(\theta)$ for the magnetic trap with strength $\lambda_{0}=$ $805 \mu \mathrm{m}$. The white dashed curve is the theoretical optimum facet angle [Eq. (5)] and the black circles are the maximum point for $z_{0} / a$. The star is the data point for the reference magnetic trap. (b) The trap frequency $\left(f_{z}\right)$ dependence with respect to the half gap $(a)$ and facet angle $(\theta)$ for $\lambda_{0}=805 \mu \mathrm{m}$. (c) The equilibrium height $\left(z_{0}\right)$ vs half gap $(a)$ with various magnetic trap strength $\left(\lambda_{0}=10 R, R\right.$, and $\left.0.1 R\right)$. (d) The critical half gap $\left(a_{c}\right)$ beyond which the confinement enhancement effect no longer applies, plotted with respect to the magnetic trap feature length $\lambda_{0}$ (at $\theta_{\max }=35.3^{\circ}$ ). The theory [Eq. (6)] fits well for $\lambda_{0} / R<2$.

facet angle $\theta_{\max }=\arccos \sqrt{2 / 3}$, which is calculated in the limit of a strong trap, applies very well to most situations where $a<a_{c}$.

\section{CONCLUSION}

In closing, compared to other existing electromagnetic trap systems known in physics [1-12], this magnetic-tip system [14] presents a simple and elementary type of magnetic trap based on conical geometry. The model that we have developed provides a rich theoretical understanding of the system that will help advance further development and applications. For example, an interesting next step is to cool down the mechanical motion of the trapped diamond towards its quantum ground state using a negative feedback cooling system and an ultrahigh vacuum environment similar to what has been done in optical traps [34-36] or other magnetic trap systems $[11,15]$. The ability to tune and achieve high frequency (e.g., up to kilohertz range) in this magnetic-tip trap to raise the quantum ground-state energy is also attractive to make the quantum regime more accessible. This magnetic-tip trap system could also be useful as inertial sensor to sense vibration or seismic activities.

\section{APPENDIX A: MAGNETIC FIELD CALCULATION}

We calculate the magnetic field along the principal axis (z) of a pair of magnetic tips as shown in Fig. 1(a). First

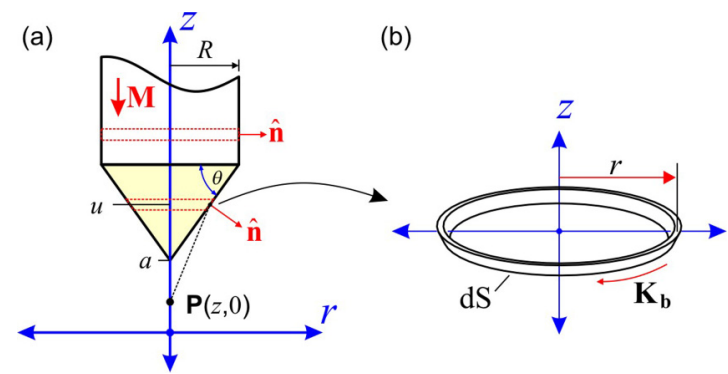

FIG. 3. (a) A magnetic-tip model. (b) Current loop on the cone surface. 
we consider a single magnetic tip as shown in Fig. 3(a). The magnetic-tip system can be modeled as a semi-infinite cylindrical section and a conical tip. The magnet has a uniform magnetization $\mathbf{M}$ with direction along the principal axis pointing toward the tip. The cylindrical section has radius $R$. The conic section has a "facet angle" $\theta$ and the tip is located at $z=a$, as shown in Fig. 3.

We divide the magnetic field calculation into two parts: the conical tip section $\left(\mathbf{B}_{\mathbf{c}}\right)$ and the cylindrical section $\left(\mathbf{B}_{\mathbf{s}}\right)$. We first consider the conical tip that has a bound surface current element at $z=u$ due to uniform magnetization $M$. The surface current forms an elemental current loop with radius $r=$ $(u-a) \cot \theta$ and surface area $d S$ which produces a magnetic field:

$$
\begin{aligned}
d \mathbf{B}_{\mathbf{c}} & =-\frac{\mu_{0}}{2} \frac{r^{2} d I}{\left(r^{2}+\Delta z^{2}\right)^{3 / 2}} \hat{\mathbf{z}} \\
& =-\frac{\mu_{0}}{2} \frac{(u-a)^{2} \cot ^{2} \theta d I}{\left[(u-a)^{2} \cot ^{2} \theta+(u-z)^{2}\right]^{3 / 2}} \hat{\mathbf{z}} .
\end{aligned}
$$

The surface current element on the conical surface can be calculated as $\mathbf{K}_{\mathbf{b}}=\mathbf{M} \times \hat{\mathbf{n}}$ where $\hat{\mathbf{n}}$ is the surface normal. The current element is given as $d I=K_{b} d S$, where $K=M \sin \theta$ and $d S=d u / \sin \theta$. As a result, the current element is equal to $d I=M d u$. The magnetic field produced by the conical tip section at point $\mathrm{P}$ is given by

$$
\mathbf{B}_{\mathbf{c}}(z)=-\frac{\mu_{0} M}{2} \int_{a}^{a+R \tan \theta} \frac{(u-a)^{2} \cot ^{2} \theta d u}{\left[(u-a)^{2} \cot ^{2} \theta+(u-z)^{2}\right]^{3 / 2}} \hat{\mathbf{z}} .
$$

The integral yields an analytical result:

$$
\begin{aligned}
\mathbf{B}_{\mathbf{c}}(z)= & -\frac{\mu_{0} M}{2}\left[\frac{(a-z) \sin ^{2} \theta \cos \theta-R \cos 2 \theta \sin \theta}{\sqrt{R^{2}+(a-z)^{2} \cos ^{2} \theta+R(a-z) \sin 2 \theta}}-\cos ^{2} \theta \sin \theta \operatorname{arctanh}(\sin \theta)\right. \\
& \left.+\cos ^{2} \theta \sin \theta \operatorname{arctanh}\left(\frac{R+(a-z) \sin \theta \cos \theta}{\sqrt{R^{2}+(a-z)^{2} \cos ^{2} \theta+R(a-z) \sin 2 \theta}}\right)-\sin ^{2} \theta\right] \hat{\mathbf{z}} .
\end{aligned}
$$

Next we calculate the magnetic field due to the cylindrical sheath, which is straightforward. Consider a bound surface current element at $z=u$ with radius $R$ and current $d I=M d u$, as shown in Fig. 3(a). The magnetic field produced by the cylindrical sheath at point $\mathrm{P}$ is given by

$$
\begin{aligned}
\mathbf{B}_{\mathbf{s}}(z) & =-\frac{\mu_{0} M}{2} \int_{a+R \tan \theta}^{\infty} \frac{R^{2} d u}{\left[R^{2}+(u-z)^{2}\right]^{3 / 2}} \hat{\mathbf{z}} \\
& =-\frac{\mu_{0} M}{2}\left[1+\frac{z-a-R \tan \theta}{\sqrt{R^{2}+(a+R \tan \theta-z)^{2}}}\right] \hat{\mathbf{z}} .
\end{aligned}
$$

The total magnetic field due to the conical tip and the sheath is $\mathbf{B}_{\mathbf{U}}(z)=\mathbf{B}_{\mathbf{c}}(z)+\mathbf{B}_{\mathbf{s}}(z)$, which is also given in Eq. (1). Finally, by exploiting the symmetry of the problem we can calculate the total magnetic field of the upper and lower magnetic tip as [Eq. (2)] $\mathbf{B}_{\mathbf{T}}(z)=\mathbf{B}_{\mathbf{U}}(z)-\mathbf{B}_{\mathbf{U}}(-z)$.

\section{APPENDIX B: OPTIMUM FACET ANGLE}

The total trap potential per unit volume of the trapped object can be approximated as a harmonic potential due to magnetic interaction plus a gravitational term: $U_{T}(z)=$ $\frac{1}{2} k_{z} z^{2}+\rho g z$, where $k_{z}$ is the spring constant per unit volume: $k_{z}=\partial^{2} U_{M} / \partial z^{2}$ and $U_{M}$ is the magnetic energy potential per unit volume given as $\chi B_{T}^{2}(z) / 2 \mu_{0}$, assuming the material is a weak diamagnet ( $\chi \ll 1$ ). Using $B_{T}$ from Eq. (2), we can obtain $k_{z}$ as shown in Eq. (4).

The optimum facet angle $\theta_{\max }$ is obtained from $d k_{z} / d \theta=$ 0 . This allows us to solve for $\theta_{\max }$ :

$$
\frac{d}{d \theta}\left[\frac{\cos ^{6} \theta(a+R \tan \theta)^{2}}{\left(R^{2}+a^{2} \cos ^{2} \theta+a R \sin 2 \theta\right)^{3}}\right]=0 .
$$

Using $\eta=a / R$ we have

$$
\left(9+4 \eta^{2}+4 \eta^{4}\right) \cos ^{4} \theta_{\max }-\left(12+8 \eta^{2}\right) \cos ^{2} \theta_{\max }+4=0 .
$$

This yields a solution for $\theta_{\max }$ as

$$
\theta_{\max }=\arccos \sqrt{\frac{2\left(3+2 \eta^{2}+2 \sqrt{2} \eta\right)}{9+4 \eta^{2}+4 \eta^{4}}}
$$

which is also shown in Eq. (5). In the limit of a small gap $(a \ll R)$, the optimum facet angle reduces to a very simple expression:

$$
\theta_{\max } \approx \arccos \sqrt{2 / 3}=35.3^{\circ}
$$

\section{APPENDIX C: FREQUENCY RATIO OF THE RADIAL AND AXIAL MODES}

We will find a relationship between the frequencies of the axial and radial oscillation modes in the magnetic-tip trap. A similar problem has been discussed by McDonald [33] for a magnetic levitation system of a diamagnet above a magnetized disk that also has a cylindrical symmetry. We summarize the key findings and extend them to our magnetic-tip trap system. The approach starts by using Maxwell's equations, $\nabla \cdot \mathbf{B}=0$ and $\nabla \times \mathbf{B}=0$, and then relating the magnetic field components $B_{r}$ and $B_{z}$ in the cylindrical coordinates.

The magnetic field components up to the second order in $r$ and $z$ can be expressed as [33]

$$
\begin{aligned}
B_{z}(r, z)= & b_{0}+b_{1}\left(z-z_{0}\right)+b_{2}\left(z-z_{0}\right)^{2} \\
& +b_{3} r+b_{4} r^{2}+b_{5} r\left(z-z_{0}\right),
\end{aligned}
$$




$$
\begin{aligned}
B_{r}(r, z)= & c_{0}+c_{1}\left(z-z_{0}\right)+c_{2}\left(z-z_{0}\right)^{2} \\
& +c_{3} r+c_{4} r^{2}+c_{5} r\left(z-z_{0}\right) .
\end{aligned}
$$

Using Maxwell equations in cylindrical coordinates,

$$
\begin{gathered}
\nabla \cdot \mathbf{B}=1 / r \partial\left(r B_{r}\right) / \partial r+\partial B_{z} / \partial z=0, \\
(\nabla \times \mathbf{B})_{\phi}=\partial B_{r} / \partial z-\partial B_{z} / \partial r=0 .
\end{gathered}
$$

Using these equations we can relate the coefficient $c_{n}$ to $b_{n}$ as detailed in Ref. [33]. To evaluate the oscillation at the equilibrium point we evaluate $B^{2}$ up to second order in $r$ and $z$ :

$$
\begin{aligned}
B^{2}= & B_{z}^{2}+B_{r}^{2}=b_{0}^{2}+2 b_{0} b_{1}\left(z-z_{0}\right) \\
& +\left(b_{1}^{2}+2 b_{0} b_{2}\right)\left(z-z_{0}\right)^{2}+\left(b_{1}^{2} / 4-b_{0} b_{2}\right) r^{2} .
\end{aligned}
$$

Now we can calculate the ratio of the radial and axial frequencies: $f_{r} / f_{z}=\sqrt{k_{r} / k_{z}}$. The spring constants for the axial mode $\left(k_{z}\right)$ and radial mode $\left(k_{r}\right)$ are given as

$$
\begin{gathered}
k_{z}=\partial^{2} U_{T} / \partial z^{2} \propto \partial^{2} B^{2}(r, z) / \partial z^{2}=b_{1}^{2}+2 b_{0} b_{2}, \\
k_{r}=\partial^{2} U_{T} / \partial r^{2} \propto \partial^{2} B^{2}(r, z) / \partial r^{2}=b_{1}^{2} / 4-b_{0} b_{2} .
\end{gathered}
$$

Then we have

$$
f_{r} / f_{z}=\sqrt{k_{r} / k_{z}}=\sqrt{\left(b_{1}^{2} / 4-b_{0} b_{2}\right) /\left(b_{1}^{2}+2 b_{0} b_{2}\right)} .
$$

For an object near the center of the trap, the field is small: $B\left(0, z_{0}\right) \sim 0$, thus $b_{0} \sim 0$ and the magnetic field is nearly linear with $z$, thus $b_{2} \sim 0$ [see Fig. 1(b)]. Therefore we have $b_{0} b_{2} \ll b_{1}^{2}$ and the last equation can be simplified to $f_{r} / f_{z}=$ $1 / 2$, which is consistent with the experimental observation.

\section{APPENDIX D: CRITICAL GAP}

In order to find the critical gap, i.e., the gap beyond which there is no more confinement enhancement effect, we can expand the magnetic energy up to the fourth order:

$$
\begin{aligned}
U_{M}(z) \approx-\frac{\chi}{8} \mu_{0} M^{2}\left(\alpha z^{2}+\beta z^{4}\right), \\
\alpha=\frac{32 R^{4} \cos ^{4} \theta(a \cos \theta+R \sin \theta)^{2}}{a^{2}\left(a^{2}+2 R^{2}+a^{2} \cos 2 \theta+2 a R \sin 2 \theta\right)^{3}}, \\
\beta=\frac{8 R^{4} \cos ^{4} \theta(a \cos \theta+R \sin \theta)}{3 a^{4}\left(a^{2}+2 R^{2}+a^{2} \cos 2 \theta+2 a R \sin 2 \theta\right)^{5}} \\
\times\left[2 a\left(60 a^{4}+47 a^{2} R^{2}+28 R^{4}\right) \cos \theta\right. \\
+a\left(60 a^{4}-77 a^{2} R^{2}-56 R^{4}\right) \cos 3 \theta \\
+a^{3}\left(12 a^{2}-65 R^{2}\right) \cos 5 \theta \\
+2 R\left(44 a^{4}+49 a^{2} R^{2}+16 R^{4}\right) \sin \theta \\
+3 a^{2} R\left(44 a^{2}+21 R^{2}\right) \sin 3 \theta \\
\left.+a^{2} R\left(44 a^{2}-35 R^{2}\right) \sin 5 \theta\right] .
\end{aligned}
$$

The equilibrium height of diamond $z_{0}$ is the solution of $\chi \mu_{0} M^{2} \alpha z_{0} / 4+\rho g=0$, so we can express $z_{0}$ in terms of $\lambda_{0}=|\chi| \mu_{0} M^{2} / \rho g$ and $\alpha: z_{0}=4 / \lambda_{0} \alpha$. The spring constant up to leading order in $z_{0}$ is

$$
k_{z}\left(\theta, z_{0}\right)=-\frac{\chi}{4} \mu_{0} M^{2}\left(\alpha+6 \beta z_{0}^{2}\right) .
$$

Confinement enhancement exists if there is an optimum angle $\theta_{\max }$ where the spring constant $k_{z}$ becomes maximum. However, there is a critical gap (or half gap $a_{c}$ ) beyond which there is no $\theta$ between $0^{\circ}$ and $90^{\circ}$ that yields maximum $k_{z}$. The condition for the critical gap is $\partial^{2} k_{z} / \partial \theta^{2}=0$, evaluated at optimum angle $\theta_{\max }=35.3^{\circ}$. The relation between $a_{c}, R$, and $\lambda_{0}$ that satisfies this condition is

$$
-\frac{7.108}{a_{c}^{2}}+\frac{16.839}{a_{c} R}+\frac{1295.442\left(R^{2}+0.021 \lambda_{0}^{2}\right)}{R^{2} \lambda_{0}^{2}}=0 .
$$

Hence, the critical gap is

$$
\frac{a_{c}}{R}=\frac{\lambda_{0}\left(\sqrt{129.9 R^{2}+3.74 \lambda_{0}^{2}}-\lambda_{0}\right)}{153.86 R^{2}+3.24 \lambda_{0}^{2}} .
$$

\section{APPENDIX E: MAGNETIC TRAP FEATURE LENGTH}

The magnetic trap feature length $\lambda_{0}$ can be considered as the characteristic length or size of a magnet where the maximum diamagnetic repulsion force balances the gravity. Larger $\lambda_{0}$ indicates a stronger magnetic trap which can levitate a diamagnetic object at longer distance. In the simplest model one could use a spherical magnet. The feature length is approximately the radius of a spherical magnet when the diamagnetic repulsion force of a diamagnetic object balances the gravity near the magnet's surface, where the maximum repulsion force occurs. To calculate this feature length, consider a small diamagnetic object (with mass density $\rho$ and magnetic susceptibility $\chi$ ) constrained in a vertical $z$ axis above a spherical magnet (with magnetization $M$ pointing to $+z$ ) centered at $z=0$. We start with the magnetic potential energy per unit volume of the diamagnetic object: $U_{M}=$ $|\chi| B^{2} / 2 \mu_{0}$, where $B$ is the magnetic field along the $z$ axis: $B=\mu_{0} m / 2 \pi z^{3}$ and $m$ is the magnetic dipole moment of the magnet.

The diamagnetic repulsion force per unit volume of the diamagnetic object is given as

$$
F_{M}^{\prime}=-\frac{\partial U_{M}}{\partial z}=\frac{3|\chi| \mu_{0} m^{2}}{4 \pi^{2} z^{7}}
$$

and when this force balances the gravity we have $F_{M}^{\prime}=\rho g$. The maximum repulsion force occurs near the surface of the magnet: $z=R$, which sets the feature length. Using $m=$ $4 \pi R^{3} M / 3$, we obtain the radius of the magnet:

$$
R=\frac{4|\chi| \mu_{0} M^{2}}{3 \rho g}
$$

As the magnetic trap feature length is used as the reference length scale and often only order-of-magnitude information is necessary, we can ignore the coefficient $4 / 3$. Thus the magnetic trap feature length is given as $\lambda_{0}=|\chi| \mu_{0} M^{2} / \rho g$. 
[1] F. M. Penning, Physica (Utrecht) 3, 873 (1936).

[2] L. S. Brown and G. Gabrielse, Rev. Mod. Phys. 58, 233 (1986).

[3] A. Ashkin, IEEE J. Quant. Elect. 6, 841 (2000).

[4] S. Chu, J. E. Bjorkholm, A. Ashkin, and A. Cable, Phys. Rev. Lett. 57, 314 (1986).

[5] S. Chu, L. Hollberg, J. E. Bjorkholm, A. Cable, and A. Ashkin, Phys. Rev. Lett. 55, 48 (1985).

[6] E. L. Raab, M. Prentiss, A. Cable, S. Chu, and D. E. Pritchard, Phys. Rev. Lett. 59, 2631 (1987).

[7] W. D. Phillips, Rev. Mod. Phys. 70, 721 (1998).

[8] M. D. Simon, L. O. Heflinger, and A. K. Geim, Am. J. Phys. 69, 702 (2001).

[9] I. F. Lyuksyutov, D. G. Naugle, and K. D. D. Rathnayaka, Appl. Phys. Lett. 85, 1817 (2004).

[10] O. Gunawan, Y. Virgus, and K. Fai Tai, Appl. Phys. Lett. 106, 062407 (2015).

[11] J. F. Hsu, P. Ji, C. W. Lewandowski, and B. D’Urso, Sci. Rep. 6, 30125 (2016).

[12] J. P. Houlton, M. L. Chen, M. D. Brubaker, K. A. Bertness, and C. T. Rogers, Rev. Sci. Instr. 89, 125107 (2018).

[13] M. D. Simon and A. K. Geim, J. Appl. Phys. 87, 6200 (2000).

[14] M. O’Brien, S. Dunn, J. Downes, and J. Twamley, Appl. Phys. Lett. 114, 053103 (2019).

[15] B. R. Slezak, C. W. Lewandowski, J.-F. Hsu, and B. R. D’Urso, New J. Phys. 20, 063028 (2018).

[16] H. J. Mamin, M. Poggio, C. L. Degen, and D. Rugar, Nat. Nanotech. 2, 301 (2007).

[17] D. Rugar, R. Budakian, H. Mamin, and B. Chui, Nature (London) 430, 329 (2004).

[18] M. T. Johnsson, G. K. Brennen, and J. Twamley, Sci. Rep. 6, 37495 (2016).

[19] T. Li, S. Kheifets, D. Medellin, and M. G. Raizen, Science 328, 1673 (2010).

[20] J. Millen, T. Deesuwan, P. Barker, and J. Anders, Nat. Nanotech. 9, 425 (2014).
[21] Z.-q. Yin, T. Li, X. Zhang, and L. M. Duan, Phys. Rev. A 88, 033614 (2013).

[22] M. Scala, M. S. Kim, G. W. Morley, P. F. Barker, and S. Bose, Phys. Rev. Lett. 111, 180403 (2013).

[23] T. M. Hoang, J. Ahn, J. Bang, and T. Li, Nat. Comm. 7, 12250 (2016).

[24] D. Kafri, J. M. Taylor, and G. J. Milburn, New J. Phys. 16, 065020 (2014).

[25] S. Bose, A. Mazumdar, G. W. Morley, H. Ulbricht, M. Toros, M. Paternostro, A. A. Geraci, P. F. Barker, M. S. Kim, and G. Milburn, Phys. Rev. Lett. 119, 240401 (2017).

[26] M. V. Berry and A. K. Geim, Eur. J. Phys. 18, 307 (1997).

[27] O. Gunawan and Y. Virgus, J. Appl. Phys. 121, 133902 (2017).

[28] The experiment in Ref. [14] uses a magnetic tip with four flat facets. We have also modeled such a system and found that our conical tip model is a very good approximation.

[29] A. Zangwill, Modern Electrodynamics (Cambridge University, Cambridge, England, 2013).

[30] J. Heremans, C. H. Olk, and D. T. Morelli, Phys. Rev. B 49, 15122 (1994).

[31] J. A. Dean, Lange's Handbook of Chemistry (McGraw-Hill, New York, 1999).

[32] A. C. Frangeskou, A. T. M. A. Rahman, L. Gines, S. Mandal, O. A. Williams, P. F. Barker, and G. W. Morley, New J. Phys. 20, 043016 (2018).

[33] K. T. McDonald, Diamagnetic levitation, http://www.hep. princeton.edu/ $\sim$ mcdonald/examples/diamagnetic.pdf (4 March 2015).

[34] J. Gieseler, B. Deutsch, R. Quidant, and L. Novotny, Phys. Rev. Lett. 109, 103603 (2012).

[35] J. Millen, P. Z. G. Fonseca, T. Mavrogordatos, T. S. Monteiro, and P. F. Barker, Phys. Rev. Lett. 114, 123602 (2015).

[36] P. Mestres, J. Berthelot, M. Spasenović, J. Gieseler, L. Novotny, and R. Quidant, Appl. Phys. Lett. 107, 151102 (2015). 